\title{
DEPENDENCE ON DIMENSION OF A CONSTANT RELATED TO THE GRÖTZSCH RING
}

\author{
GLEN D. ANDERSON
}

ABstract. For the constant $\lambda_{n}=\lim _{a \rightarrow 0}\left(\bmod R_{G, n}(a)+\log a\right)$ associated with the Grötzsch extremal ring $R_{G, n}$ in euclidean $n$-space, we obtain the limit $\lim _{n \rightarrow \infty} \lambda_{n}^{1 / n}=e$.

1. Definitions and notation. By a ring $R$ is meant a domain in euclidean $n$ space $R^{n}$ whose complement consists of two components $C_{0}$ and $C_{1}$, where $C_{0}$ is bounded. We let $B_{0}=\partial C_{0}$ and $B_{1}=\partial C_{1}$ be the boundary components of $R$. The conformal capacity (cf. [8]) of $R$ is

$$
\operatorname{cap} R=\inf _{\varphi} \int_{R}|\nabla \varphi|^{n} d \omega
$$

where $\nabla$ denotes the gradient, and where the infimum is taken over all realvalued $C^{1}$ functions $\varphi$ in $R$ with boundary values 0 on $B_{0}$ and 1 on $B_{1}$. Then the modulus of the ring $R$ is defined by

$$
\bmod R=\left(\sigma_{n-1} / \operatorname{cap} R\right)^{1 /(n-1)},
$$

where for each positive integer $p$ we let $\sigma_{p}$ denote the $p$-dimensional measure of the unit sphere $S^{p}=\left\{\left(x_{1}, \ldots, x_{p+1}\right): \sum_{j=1}^{p+1} x_{j}^{2}=1\right\}$. Then

$$
\sigma_{p}=2 \pi^{(p+1) / 2} \Gamma((p+1) / 2)^{-1}
$$

(cf. [7], [9]), where $\Gamma$ denotes the classical Gamma function; moreover, the relation

$$
\int_{0}^{\pi / 2} \cos ^{p} u d u=\frac{\sigma_{p+1}}{2 \sigma_{p}}
$$

holds for each positive integer $p$.

2. Background of problem and statement of result. Let $R_{G, n}(a)$ denote the $n$ dimensional Grötzsch ring, that is, the ring whose complementary components are

$$
C_{0}=\left\{\left(x_{1}, \ldots, x_{n}\right): 0 \leqslant x_{1} \leqslant a, x_{j}=0,2 \leqslant j \leqslant n\right\}
$$

and

Received by the editors June 2, 1975.

AMS (MOS) subject classifications (1970). Primary 30A60.

Copyright $\odot 1977$, American Mathematical Society 


$$
C_{1}=\left\{\left(x_{1}, \ldots, x_{n}\right): \sum_{j=1}^{n} x_{j}^{2} \geqslant 1\right\} .
$$

In [5] Gehring proved that $\bmod R_{G, 3}(a)+\log a$ is monotone decreasing in the interval $0<a<1$ and he obtained bounds $4 \leqslant \lambda_{3} \leqslant 12.4 \cdots$, where $\log \lambda_{3}$ $=\lim _{a \rightarrow 0}\left(\bmod R_{G, 3}(a)+\log a\right)$. Using analogous methods in higher dimensions, Caraman [3] and Ikoma [7] have shown that the limit

$$
\log \lambda_{n}=\lim _{a \rightarrow 0}\left(\bmod R_{G, n}(a)+\log a\right)
$$

exists for each $n \geqslant 3$. Bounds for $\lambda_{n}$ have been obtained by Gehring [5], [6], Caraman [3], Ikoma [7], and Anderson [2] (cf. [1]).

In the present paper we determine the order of growth of $\lambda_{n}$ for large $n$ by establishing the following result.

THEOREM. $\lim _{n \rightarrow \infty} \lambda_{n}^{1 / n}=e$, where $e$ is the base of Naperian logarithms.

3. Proof of Theorem. First, by the work of Caraman [3] and Ikoma [7] we know that

$$
\log \frac{\lambda_{n}}{4} \leqslant I_{n}=\int_{1}^{\infty}\left[\left(\frac{r^{2}+1}{r^{2}-1}\right)^{(n-2) /(n-1)}-1\right] \frac{d r}{r} .
$$

Under the change of variable $t=\left(r^{2}+1\right) /\left(r^{2}-1\right)$ the integral $I_{n}$ in (2) reduces to

$$
I_{n}=\int_{1}^{\infty} \frac{t^{(n-2) /(n-1)}-1}{t^{2}-1} d t .
$$

Making use of the fact that $\left(t^{2}-1\right)^{-1}=t^{-2}+t^{-2}\left(t^{2}-1\right)^{-1}$, one may rewrite $I_{n}$ as the sum of two integrals. The first of these is easy to evaluate exactly as $n-2$, and by means of the Monotone Convergence Theorem it is easy to see that the second increases to the limit $1-\log 2$. This procedure, together with (2), yields the result

$$
\log \frac{\lambda_{n}}{4}-n \leqslant \lim _{n \rightarrow \infty}\left(I_{n}-n\right)=-1-\log 2
$$

from which the upper bound $\lambda_{n}^{1 / n} \leqslant e$ is easily derived (cf. [4, Lecture 9, Theorem 1]).

On the other hand, it was determined in [2] that

(5) $\log \frac{\lambda_{n}}{4} \geqslant \int_{0}^{\infty}\left[\left(\frac{2 \sigma_{n-2}}{\sigma_{n-1}} \int_{0}^{\pi / 2}\left(\sec ^{2} u+\operatorname{csch}^{2} v\right)^{(2-n) / 2} d u\right)^{1 /(1-n)}-1\right] d v$

By making the change of variable $t=\operatorname{coth} v$ in (5) and combining the resulting inequality with (1), (2), and (3), we achieve

$$
0 \geqslant \log \frac{\lambda_{n}}{4}-I_{n} \geqslant \int_{1}^{\infty} \Phi_{n}(t) d t
$$

where 
(7) $\Phi_{n}(t)=\frac{t^{(n-2) /(n-1)}}{t^{2}-1}\left[\left(\frac{2 \sigma_{n-2}}{\sigma_{n-1}} \int_{0}^{\pi / 2}\left(1+\frac{\tan ^{2} u}{t^{2}}\right)^{(2-n) / 2} d u\right)^{1 /(1-n)}-1\right]$.

We consider first the integral of $\Phi_{n}(t)$ over the finite interval $(1, M)$. First, using (1), it is convenient to rewrite (7) in the form

$$
\Phi_{n}(t)=\frac{t^{(n-2) /(n-1)}}{t^{2}-1}\left[\left(\frac{\|\cos u\|_{n-2}}{\left\|\left(1+t^{-2} \tan ^{2} u\right)^{-1 / 2}\right\|_{n-2}}\right)^{(n-2) /(n-1)}-1\right]
$$

where $\|f\|_{p}$ denotes the $L_{p}[0, \pi / 2]$-norm of $f$. Since the functions cos $u$ and $\left(1+t^{-2} \tan ^{2} u\right)^{-1 / 2}$ are continuous on $[0, \pi / 2]$, a well-known result in the theory of $L_{p}$ spaces gives

(9) $\lim _{n \rightarrow \infty} \frac{\|\cos u\|_{n-2}}{\left\|\left(1+t^{-2} \tan ^{2} u\right)^{-1 / 2}\right\|_{n-2}}=\frac{\max _{0 \leqslant u \leqslant \pi / 2}|\cos u|}{\max _{0 \leqslant u \leqslant \pi / 2}\left|1+t^{-2} \tan ^{2} u\right|^{-1 / 2}}=1$.

From (8) and (9) it follows that for each fixed $t \in(1, M)$,

$$
\lim _{n \rightarrow \infty} \Phi_{n}(t)=0
$$

Next, in view of (8) it is easy to see that

$$
0>\Phi_{n}(t) \geqslant t^{(n-2) /(n-1)}\left(t^{(2-n) /(n-1)}-1\right) /\left(t^{2}-1\right) \geqslant-1 /(1+t) .
$$

This and (10) allow us to invoke the Dominated Convergence Theorem to conclude that

$$
\lim _{n \rightarrow \infty} \int_{1}^{M} \Phi_{n}(t) d t=0
$$

for each fixed $M \in(1, \infty)$.

Next, using the fact that $t^{2} /\left(t^{2}-1\right)$ is decreasing for $t>1$ and that $\Phi_{n}(t) \leqslant 0$, we may write

$$
\int_{M}^{\infty} \Phi_{n}(t) d t \geqslant \frac{M^{2}}{M^{2}-1}\left[\left(\frac{\|\cos u\|_{n-2}}{\|1\|_{n-2}}\right)^{(n-2) /(n-1)}-1\right] \int_{M}^{\infty} t^{(n-2) /(n-1)-2} d t
$$

Thus

$\frac{1}{n} \int_{M}^{\infty} \Phi_{n}(t) d t \geqslant \frac{M^{2}}{M^{2}-1}\left(1-\frac{1}{n}\right) M^{1 /(1-n)}\left[\left(\frac{2}{\pi}\right)^{1 /(n-1)}\|\cos u\|_{n-2}^{(n-2) /(n-1)}-1\right]$,

and the same argument as that leading to (10) shows that

$$
\liminf _{n \rightarrow \infty} \frac{1}{n} \int_{M}^{\infty} \Phi_{n}(t) d t \geqslant 0 .
$$

But because of (11), (6), and (4), this implies the statement

$$
\liminf _{n \rightarrow \infty} \frac{1}{n} \log \lambda_{n} \geqslant 1
$$


Finally, the Theorem follows from (2) and (12).

\section{REFERENCES}

1. G. D. Anderson, Symmetrization and extremal rings in space, Ann. Acad. Sci. Fenn. Ser. AI No. 438 (1969), 1-24. MR 41 \# 465.

2. - Extremal rings in n-space for fixed and varying n, Ann. Acad. Sci. Fenn. Ser. AI No. 575 (1974), 1-21.

3. P. Caraman, On the equivalence of the definitions of the n-dimensional quasiconformal homeomorphisms (QCfH), Rev. Roumaine Math. Pures Appl. 12 (1967), 889-943. MR 37 \# 409.

4. F. W. Gehring, Lectures on quasiconformal mappings, Institut Mittag-Leffler, 1972 (unpublished).

5. - Symmetrization of rings in space, Trans. Amer. Math. Soc. 101 (1961), 499-519. MR 24 \# A2677.

6. Inequalities for condensers, hyperbolic capacity, and extremal lengths, Michigan Math. J. 18 (1971), 1-20. MR 44 \#2915.

7. K. Ikoma, An estimate for the modulus of the Grötzsch ring in n-space, Bull. Yamagata Univ. Natur. Sci. 6 (1967), no. 4, 395-400. MR 43 \# 508.

8. C. Loewner, On the conformal capacity in space, J. Math. Mech. 8 (1959), 411-414. MR 21 \#3538.

9. J. Väisälä, Lectures on n-dimensional quasiconformal mappings, Lecture Notes in Math., vol. 229, Springer-Verlag, Berlin, 1971.

Department of Mathematics, Michigan State University, East Lansing, Michigan 48824 